\title{
On the Introduction of Lumped Inductors for Optimizing UHF Band AMCs in Terms of Size and Angular Stability
}

\author{
H. F. Álvarez, M. E. de Cos and F. Las-Heras \\ Area de Teoría de la Señal y Comunicaciones. Dpt. Ingeniería Eléctrica, Universidad de Oviedo. \\ Edificio Polivalente, Mod. 8. E-33203 Gijón, Asturias, Spain \\ hfernandezltsc.uniovi.es
}

Keywords: Angular Stability, Metasurfaces, Artificial Magnetic Conductor, Reflection Coefficient, Lumped inductors.

\begin{abstract}
A novel method to tailor the angular stability of Artificial Magnetic Conductors (AMCs) through the introduction of lumped inductors is introduced in this contribution. The AMC is designed, considering the common restrictions that a designer has to meet, to resonate at $406 \mathrm{MHz}$ (band widely used for Maritime Rescue System). A complete angular stability study is carried out and interesting conclusions concerning operational bandwidth, unit-cell miniaturization and angular stability improvement are drawn. Moreover, the choice of the optimum lumped inductors value is discussed.
\end{abstract}

\section{Introduction}

Artificial Magnetic Conductors (AMCs) are metasurface structures (2D metamaterials) tailored to implement Perfect Magnetic Conductor behaviour. i.e. to exhibit in-phase reflection $(\Gamma=+1)$ in a limited frequency band. Thus, they are commonly characterized using both the magnitude and phase of their reflection coefficient. The later varies from $+180^{\circ}$ to $180^{\circ}$, crossing $0^{\circ}$ at their resonance frequency. The AMC operating bandwidth comprises the frequencies in the $\pm 90^{\circ}$ variation range. AMCs exhibit high impedance surface at their resonance frequency [1]-[5] and are generally implemented as a Frequency Selective Surface (FSS) on a grounded dielectric.

AMCs are used in a wide variety of applications such as Radar Cross Section (RCS) reduction behaving as a Radar Absorbing Materials (RAM) [6]-[7], Radio Frequency Identification (RFID) [8] and as antennas' ground plane to improve their radiation properties (gain, efficiency, bandwidth...) [9]-[14]. In addition to improving the radiation properties of antennas, they can reduce the Specific Absorption Rate (SAR) which is highly desirable for applications using wearable devices [15].

In actual applications the available space to place the AMC is restricted. Therefore, the reduction of the AMC's unit-cell in order to be able to arrange as much unit-cells as possible in that fixed space is critical. In fact, this is even more critical at low frequencies where the size of these unit-cells tend to be very large. Several techniques to reduce the unit-cell size have been reported in the literature. Some of them are the use of metallized convoluted arrays of spirals [10] and the introduction of lumped components [9], [12]. On the other hand, when an AMC is used as antenna's ground plane, as it is placed in the close proximity of the antenna, different modes impinges the AMC at different angles and hence, the metasurface should be angularly stable, about the desired AMC operation band, if the radiation properties of the antenna are wanted to be improved [5].

Consequently, this paper aims to study the potential improvement of UHF AMCs in terms of size reduction and angular stability through a novel method consisting in the introduction of lumped inductors in the unit-cell metallization geometry. In the literature there are several works devoted to unit-cell miniaturization mostly using lumped capacitors. To the authors knowledge there are no previous works considering the angular stability.

\section{AMC design}

The angular stability under oblique incidence depends on: the geometry of the unit-cell's metallization, the thickness of the dielectric and its relative dielectric permittivity and the unitcell size (periodicity) compared to the dielectric substrate thickness [4]-[5].

To perform a study concerning the introduction of lumped inductors on the unit-cells metallization, several of these factors are going to be fixed bearing in mind the usual working manner of a designer. i.e., for a given operating frequency (depending on the intended application) and using a given substrate, the aim is to design an AMC being as angularly stable as possible, with suitable bandwidth and with reduced unit-cells size.

At low operating frequencies, such as the UHF ones targeted in this work, the requirements of size reduction and suitable bandwidth are specially challenging, since the bandwidth decreases and the unit-cell size increases with the resonance frequency reduction.

It is well-known from previous studies that a trade-off solution has to be adopted for the dielectric substrate thickness and the relative dielectric permittivity values, to obtain a suitable AMC operating bandwidth and angular stability for an intended application. The AMC operating bandwidth widens as the substrate becomes thicker, but the angular stability worsens. Moreover, the angular stability 
under oblique incidence gets better as the relative dielectric permittivity rises up, but the AMC operating bandwidth becomes narrower.

In this work the $406 \mathrm{MHz}$ frequency band is chosen for the AMCs operation since it is widely used for Maritime Rescue Systems, such as Cospass Sarsat, and also for some medical applications. The RO3010 dielectric substrate $\left(\varepsilon_{\mathrm{r}}=11.2\right.$ and $\tan \delta<0.0022$ ) with the same thickness $h=10.24 \mathrm{~mm}$ and $18 \mu \mathrm{m}$ metallization thickness is used for all the structures under study. With these values an AMC operating bandwidth of about 3\% could be obtained and the attention is focused on potential enhancement obtained from the introduction of lumped inductors on the metallization geometry, in terms of angular stability and unit-cell size reduction.

From the results obtained in previous works [18]-[20] it seems that unit-cells based on hexagonal geometries provide better angular stability than those based on square geometries for similar or even wider AMC operation bandwidth at similar resonance frequency.

Moreover, the angular stability of simple hexagonal and square shaped unit-cells with basic geometries: patches and loops was analyzed in [21] for TE and TM polarized waves. The parameter $\mathrm{g}$ (and so the distance between unit-cells $2 \mathrm{~g}$ ) has a great influence on the angular stability of both the patch-based and the loop-based unit-cells, so that the angular stability increases for higher $\mathrm{g}$ values. From a certain $\mathrm{g}$ value, the hexagonal-shaped unit-cells are more stable than the square-shaped unit-cells for both loop-based and patch-based metallizations. Furthermore, loop-based unit-cells are much smaller in size (about one third) than patch-based unit-cells for the same value of $\mathrm{g}$, and for the same resonance frequency.

Taking into account the previous ideas, hexagonal loop-based unit-cells are selected for this study.

\subsection{Unit-cell geometry and simulation Setup description}

For the hexagonal loop unit-cells under study (See Fig.1 (a) and (d)), $W$ refers to the metallization dimension, $g$ is the gap distance from the metallization to the edge of the unit-cell (i.e. the gap-distance between unit-cells is $2 g$ ), and $P$ is the periodicity. Besides, $l$ is the width of the loops metallization.

The width of the metallic strips, $l=1.45 \mathrm{~mm}$, has been chosen so that 0805 sized lumped inductors could be introduced one at each side of the metallization geometry for comparison.

To determine the AMC operating bandwidth full-wave Finite Element Method (FEM) based electromagnetic simulations are carried out using HFSS [16] along with the Bloch-Floquet theory. Periodic boundary conditions (PBC) are set to a unitcell resembling an infinite structure to simulate its reflection coefficient for a uniform incident plane wave [18] .

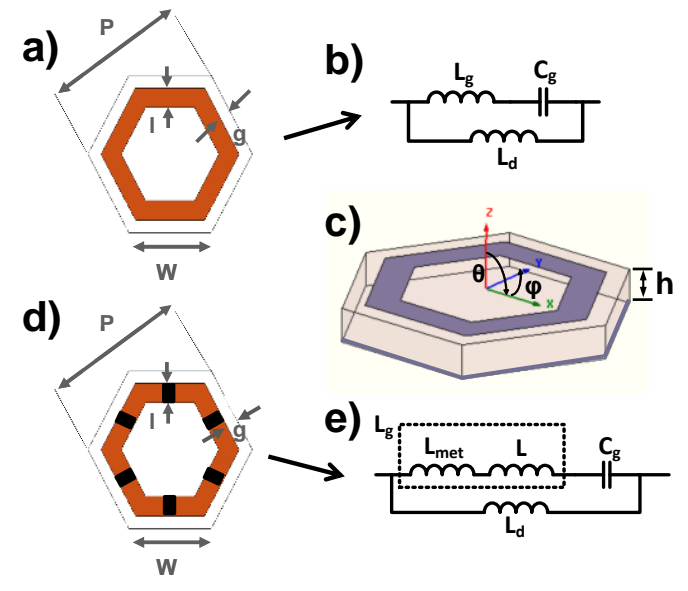

Fig. 1. (a) Unit-cell geometry (top view); (b) equivalent circuit model for loop-based unit-cells; (c) coordinate system for angular stability analysis; (d)Unit-cell geometry with lumped inductors (top view); (e) equivalent circuit model for loop-based unit-cells with lumped inductors .

\subsection{Angular stability analysis}

The methodology to study the angular stability involves: varying the polarization angle of the incident field $(\varphi)$ from $0^{\circ}$ to $90^{\circ}$ in steps of $15^{\circ}$, while varying the incidence angle $(\theta)$ from $0^{\circ}$ to $60^{\circ}$, also in steps of $15^{\circ}$, for each $\varphi$ angle. Both TE and TM polarized incident plane waves are taken into account. The angular stability of the AMC is determined not only in terms of both the absolute $(\Delta \mathrm{f})$ and relative $(\Delta \mathrm{f}(\%))$ resonance frequency deviation [17] but also considering the absolute stable bandwidth (Bs) which comprises the frequencies for which the periodic surfaces behave as AMC and its relative value $(\mathrm{Bs}(\%))[21]$.

For comparison regarding angular stability, the worst-case is considered from the complete angular analysis, i.e. the largest $\Delta \mathrm{f}$ and the narrowest Bs are selected.

\section{Results}

The dimensions of the hexagonal loop-based unit-cell (see Fig. 1 (a)) have been firstly determined through simulation, so that it exhibits AMC behaviour at $406 \mathrm{MHz}$ with a suitable bandwidth of about $2-3 \%$. For the dielectric thickness, $h=10.24 \mathrm{~mm}$, and a gap between unit-cells, $2 g=4 \mathrm{~mm}$, a periodicity $P=67.2 \mathrm{~mm}$ and a metallization dimension $W=36.5 \mathrm{~mm}$ are obtained.

Then, in order to achieve certain unit-cell size reduction while preserving the resonance frequency and the bandwidth, lumped inductors, $L$, are introduced (see Fig.1 (c)), while keeping fixed the dielectric thickness and the gap between unit-cells. Commercially available 0805 sized inductor values with proper Self Resonance Frequency (SRF) are considered to this aim.

Table 1 depicts the resonance frequency $\left(\mathrm{f}_{\mathrm{r}}\right)$ AMC operating bandwidth $(\mathrm{Bw})$ and dimensions for the analyzed unit-cells under normal incidence conditions. It can be observed that, 
as expected, the unit-cell size decreases for increasing values of the introduced lumped inductor, $L$. However, it is remarkable that the AMC operating bandwidth widens for lower $\mathrm{L}$ values, up to $8.2 \mathrm{nH}$, then similar values as for the unit-cell without inductors are obtained for $\mathrm{L}=10-12 \mathrm{nH}$, and finally it narrows for higher $\mathrm{L}$ values. This can be also observed in Fig. 2, showing the reflection coefficient phase variation with frequency for the metasurface under consideration. Thus, a designer has to adopt a trade-off solution concerning size reduction versus bandwidth.

\begin{tabular}{|l|l|l|l|l|l|l|}
\hline \hline $\mathrm{L}(\mathrm{nH})$ & $\begin{array}{l}\mathrm{BW} \\
(\mathrm{MHz})\end{array}$ & $\begin{array}{l}\mathrm{Bw} \\
(\%)\end{array}$ & $\begin{array}{l}\text { fr } \\
(\mathrm{MHz})\end{array}$ & $\begin{array}{l}\mathrm{W} \\
(\mathrm{mm})\end{array}$ & $\begin{array}{l}\mathrm{P} \\
(\mathrm{mm})\end{array}$ & $\begin{array}{c}\text { Area } \\
\left(\mathrm{cm}^{2}\right)\end{array}$ \\
\hline- & 11.6 & 2.7 & 405.5 & 36.5 & 67.2 & 39.1 \\
\hline 3.3 & 13.1 & 3.2 & 406 & 33.0 & 61.2 & 32.4 \\
\hline 6.8 & 12.2 & 3.0 & 407 & 30.8 & 57.4 & 28.5 \\
\hline 8.2 & 12.0 & 2.9 & 405.5 & 30.0 & 55.9 & 27.1 \\
\hline 10 & 11.0 & 2.7 & 406.7 & 29.2 & 54.6 & 25.8 \\
\hline 12 & 10.7 & 2.6 & 409.1 & 28.0 & 52.5 & 23.9 \\
\hline 15 & 9.7 & 2.4 & 406 & 26.6 & 50.1 & 21.7 \\
\hline 47 & 4.5 & 1.1 & 406 & 16.4 & 32.5 & 9.1 \\
\hline \hline
\end{tabular}

Table 1: AMC Bandwidth. Resonance frequency and dimensions for the unit-cells under study.

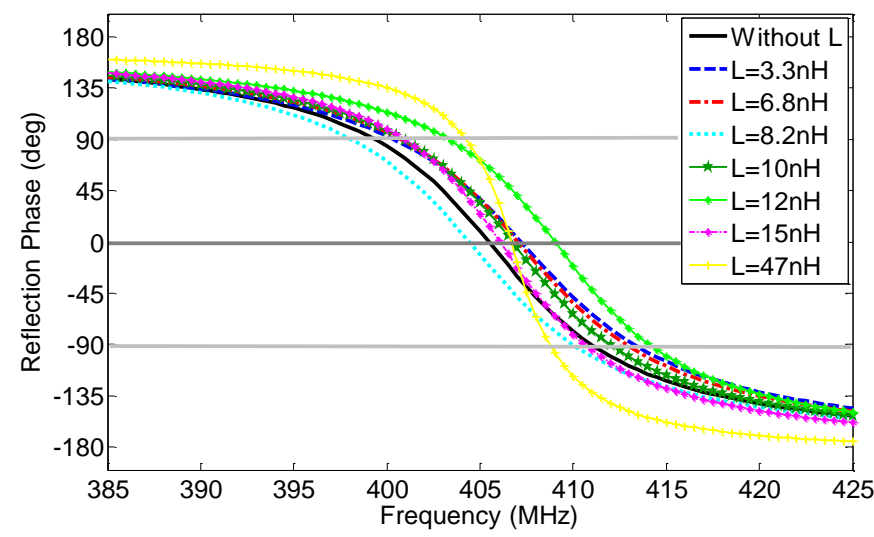

Fig. 2. Reflection coefficient phase for hexagonal loop-based unit-cell with different introduced Lumped inductor values for TE-polarized incident plane-waves.

To ensure the AMC behaviour of the metasurface it is necesarry to consider not only the reflection coefficient phase but also its amplitude. From Fig. 3 it can be observed that similar amplitude values, around 0.8 due to the dielectric substrate losses, are obtained for the unit-cell without inductors, and for the majority of the $\mathrm{L}$ values under consideration. However, for very high inductor values, as $\mathrm{L}=47 \mathrm{nH}$, the reflection coefficient amplitude dramatically decreases, so that the metasurface cannot be considered as an AMC. This agrees with the obtained surface impedance Zs versus frequency depicted in Fig 4. High $\mathrm{Zs}$ values corresponding to AMC behavior are exhibited for the metasurface up to $\mathrm{L}=15 \mathrm{nH}$, whereas much a lower $\mathrm{Zs}$ value is obtained for $\mathrm{L}=47 \mathrm{nH}$.

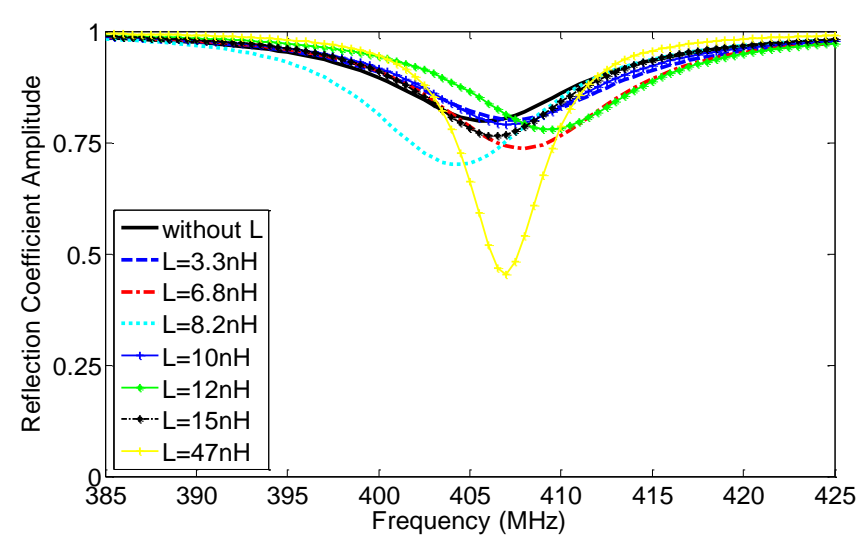

Fig. 3. Reflection coefficient amplitude for hexagonal loop-based unit-cell with different introduced Lumped inductor values for TE-polarized incident plane-waves.

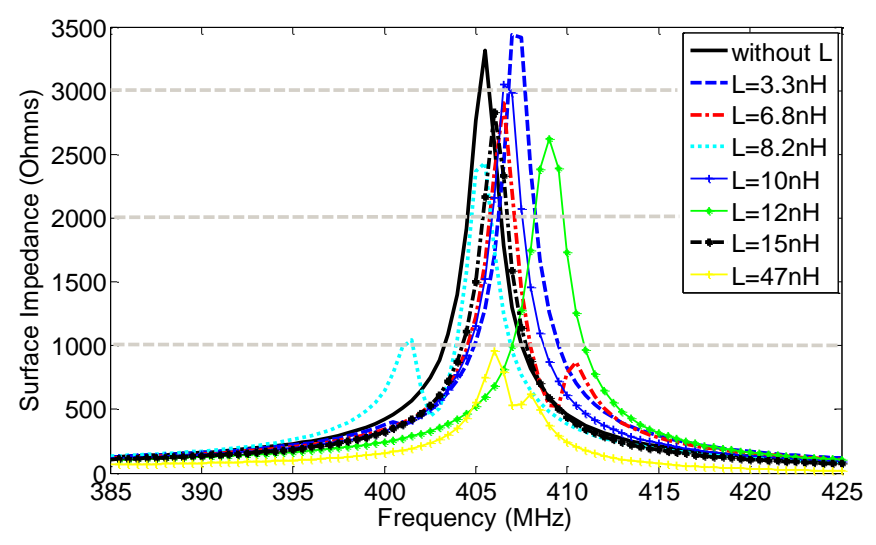

Fig. 4. Surface Impedance for hexagonal loop-based unit-cell with different introduced Lumped inductor values for TE-polarized incident planewaves.

Table 2 shows the worst-case results obtained for the complete angular stability study conducted according to the procedure described in section 2.2.

It can be observed that the unit-cell without lumped inductors is already very stable, for both TE and TM polarizations, due to the hexagonal loop-based selected geometry, but its size is large.

It is noticeable that for lower lumped inductor values (up to $\mathrm{L}=8.2 \mathrm{nH}$ ), the angular stability worsens, not only in terms of resonance frequency deviation $\Delta \mathrm{f}$ but also concerning the stable bandwidth $\mathrm{Bs}$, for both $\mathrm{TE}$ and $\mathrm{TM}$ polarizations. However, for $\mathrm{L}=10-12 \mathrm{nH}$, similar angular stability as for the unit-cell without lumped inductors can be obtained, while reducing the size more than one third, from $39.1 \mathrm{~cm}^{2}$ to $23.9 \mathrm{~cm}^{2}$ (see Table 1). In fact, the resonance frequency deviation $\Delta \mathrm{f}$ is improved with $\mathrm{L}=12 \mathrm{nH}$. For higher inductor values, $\mathrm{L}=15 \mathrm{nH}$ and $\mathrm{L}=47 \mathrm{nH}$, the angular stability worsens. So, once again it seems that the optimum $\mathrm{L}$ value is in the range of $10-12 \mathrm{nH}$ considering suitable bandwidth, sizereduction and angular stability. 


\begin{tabular}{|l|l|l|l|l|l|l|l|l|}
\hline \hline \multirow{3}{*}{$\mathbf{L}(\mathbf{n H})$} & \multicolumn{9}{|c|}{ TE } & \multicolumn{4}{c|}{ TM } \\
\cline { 2 - 9 } & $\begin{array}{c}\Delta \mathrm{f} \\
(\mathrm{MHz})\end{array}$ & $\begin{array}{c}\Delta \mathrm{f} \\
(\%)\end{array}$ & $\begin{array}{c}\mathrm{Bs} \\
(\mathrm{MHz})\end{array}$ & $\begin{array}{c}\text { Bs } \\
(\%)\end{array}$ & $\begin{array}{c}\Delta \mathrm{f} \\
(\mathrm{MHz})\end{array}$ & $\begin{array}{c}\Delta \mathrm{f} \\
(\%)\end{array}$ & $\begin{array}{c}\mathrm{Bs} \\
(\mathrm{MHz})\end{array}$ & $\begin{array}{c}\mathrm{Bs} \\
(\%)\end{array}$ \\
\hline- & 7.9 & 1.9 & 1.0 & 0.2 & 8.1 & 1.9 & 8.5 & 2.1 \\
\hline 3.3 & 15.8 & 3.8 & 0.0 & 0.0 & 17.0 & 4.2 & 0.0 & 0.0 \\
\hline 6.8 & 12.4 & 3.0 & 0.0 & 0.0 & 13.5 & 3.2 & 3.7 & 0.9 \\
\hline 8.2 & 16.1 & 4.0 & 0.0 & 0.0 & 12.5 & 3.1 & 1.6 & 0.4 \\
\hline 10 & 7.2 & 1.8 & 1.0 & 0.2 & 10.3 & 2.5 & 3.1 & 0.8 \\
\hline 12 & 7.3 & 1.8 & 0.3 & 0.1 & 8.0 & 1.9 & 4.7 & 1.2 \\
\hline 15 & 10.5 & 2.6 & 0.0 & 0.0 & 9.7 & 2.4 & 2.2 & 0.6 \\
\hline 47 & 18.5 & 4.6 & 0.0 & 0.0 & 20.8 & 5.2 & 0.0 & 0.0 \\
\hline \hline
\end{tabular}

Table 2: Resonance frequency deviation and stable bandwidth for TE and TM polarized incident plane-waves.

\subsection{Discussion}

A simplified model can be used to explain the possible reasons for the behaviour described in the previous section [21]. Since an AMC can be considered a grounded metallodielectric FSS and provided that the periodicity of the unitcells under study is electrically small, the surface impedance seen by an obliquely incident plane wave $(Z s)$ can be obtained as a parallel connection of the effective grid impedance, corresponding to the metallo-dielectric array $(Z g)$, and the input impedance of the grounded dielectric substrate $(Z d)$. The surface impedances for TE and TM polarizations, $\mathrm{Zs}{ }^{\mathrm{TE}}$ and $\mathrm{Zs}^{\mathrm{TM}}$, will lead to their corresponding reflection coefficients, $\Gamma^{\mathrm{TE}}$ and $\Gamma^{\mathrm{TM}}$

$$
\begin{gathered}
\Gamma^{\mathrm{TE}}=\frac{Z_{s}^{T E} \cos (\theta)-\eta_{0}}{Z_{s}^{T E} \cos (\theta)+\eta_{0}} \\
\Gamma^{\mathrm{TM}}=\frac{Z_{s}^{T M}-\eta_{0} \cos (\theta)}{Z_{s}^{T M}+\eta_{0} \cos (\theta)}
\end{gathered}
$$

where $\eta_{0}$ is the free space impedance and

For the loop-based unit-cell under study, the grid impedance, $Z g$, is a series connection of a grid inductance $(\mathrm{Lg})$ and a grid capacitance $(\mathrm{Cg})$ as it has been explained in [23]. The grounded dielectric slab can be modelled as an inductance $(L d)$ provided that its thickness is electrically small at the operating frequency. It is assumed that $L g$ and $C g$ do not depend on the incidence angle $\theta . \quad \mathrm{Ld}=12.8 \mathrm{nH}$ is obtained for all the unit-cells under study due to the considered dielectric thickness $(\mathrm{h}=10.24 \mathrm{~mm})$.

According to Table 2, for lumped inductor values lower than the dielectric slab equivalent inductance, i.e $\mathrm{L}<\mathrm{Ld}$, the angular stability worsens, as it can be observed for L up to $\mathrm{L}=8.2 \mathrm{nH}$. This can be attributable to a resulting $\mathrm{Zg}$ increasing the variation of $\mathrm{Zs}$ with the incident and polarization angles. However, the most remarkable result is that for $\mathrm{L} \approx \mathrm{Ld}$, the miniaturized unit-cell preserves the angular stability of the unit-cell without lumped inductor while reducing the size more than one third. This can be observed for $\mathrm{L}=10-12 \mathrm{nH}$, being $\mathrm{L}=12 \mathrm{nH}$ the optimum value since is the closer one to Ld. It seems that the resulting $\mathrm{Zg}$ leads to similar $\mathrm{Zs}$ angular sensitivity. Finally, for L> Ld the angular stability worsens compare to the unit-cell without lumped inductors. This is the case of $\mathrm{L}=15 \mathrm{nH}$ and it is especially noticeable for $\mathrm{L}>>\mathrm{Ld}$ as for $\mathrm{L}=47 \mathrm{nH}$, which leads to a very unstable resulting unitcell. The resulting Zg increases the Zs angular sensitivity.

Thus, it can be concluded that unit-cell size-reduction is possible while preserving the angular stability and AMC operating bandwidth by introducing lumped inductors of a proper value, $\mathrm{L} \approx \mathrm{Ld}$.

AMC's with smaller unit-cells and resonating at frequencies close to the one presented here have been presented in [9], [10], [13]-[15]. However, these AMCs exhibit smaller bandwidth than the one presented in this contribution. Moreover, the dielectric used in [9], [13]-[15] is FR4, which has higher dielectric losses than RO3010. On the other hand, the AMCs presented in the mentioned papers are based on square patch unit-cells which, as it was demonstrated in [21], are not as angularly stable as the hexagonal loop-based ones. Therefore, the AMC presented in this contribution not only miniaturize the unit-cell but also maintains the angular stability of the initially larger one (AMC without inductors).

From the obtained results through this study, a design methodology can be extracted: once the dielectric thickness is fixed for the aimed resonance frequency, AMC operating bandwidth and angular stability, the corresponding Ld can be calculated and so the optimum lumped inductor value which will lead to the optimum miniaturized unit-cell. A designer has of course to consider the commercially available dielectric substrate thicknesses along with the lumped inductor ones.

\section{Conclusions}

This work has analyzed the potential enhancements concerning the angular stabilization and the size-reduction of an hexagonal loop-based AMC, through a novel approach entailing the use of lumped inductances.

As a reward from the lumped inductances introduction, unit-cell size-reduction is achieved while preserving the angular stability and AMC operating bandwidth.

A simple design methodology and insight to calculate the optimum lumped inductor value is presented.

This work has been focused on UHF Band AMCs due to the size reduction and suitable bandwidth challenges. However the methodology can be also applied not only for lower frequency bands, but also to higher ones, provided that the resulting unit-cell size makes sense in terms of the manufacturing constraints and the lumped inductors size versus the metallization one.

The authors continue working on the application of these results for antennas performance improvement, due to the limitations for the proper angular stability measurement itself in anechoic chamber [24]. 


\section{Acknowledgements}

This work has been supported by the Gobierno del Principado de Asturias/FEDER under project GRUPIN14114 and by the Ministerio de Economía y Competitividad under project TEC2014-54005-P (MIRIIEM).

\section{References}

[1] D. Sievenpiper, Lijun Zhang, R. Broas, N. Alexopolous and E. Yablonovitch, "High-impedance electromagnetic surfaces with a forbidden frequency band", IEEE Transactions on Microwave Theory and Techniques, vol. 47, no. 11, pp. 2059-2074, (1999).

[2] A. Feresidis, G. Goussetis, and J. Vardaxoglou, "Metallodielectric arrays without vias as artificial magnetic conductors and electromagnetic band gap surfaces," IEEE Antennas and Propagation Society Symposium, (2004).

[3] K. Agarwal and Y.-X. Guo, "Interaction of electromagnetic waves with humans in wearable and biomedical implant antennas," 2015 Asia-Pacific Symposium on Electromagnetic Compatibility (APEMC), (2015).

[4] O. Luukkonen, F. Costa, C.R. Simovski, A. Monorchio and S.A. Tretyakov, "A Thin Electromagnetic Absorber for Wide Incidence Angles and Both Polarizations," IEEE Trans. on Antennas and Propagation, vol. 57, no. 10, pp.3119-3125, Oct. 2009

[5] C.R. Simovski, P.D. Maagt, I.V. Melchakova, "Highimpedance surfaces having stable resonance with respect to polarization and incidence angle", IEEE Trans.on Ant. and Prop., Vol. 53,No.3,908-914, March 2005.

[6] J. C. Iriarte Galarregui, A. Tellechea Pereda, J. L. M. de Falcón, I. Ederra, R. Gonzalo and P. de Maagt, "Broadband Radar Cross-Section Reduction Using AMC Technology," in IEEE Transactions on Antennas and Propagation, vol. 61, no. 12, pp. 6136-6143, Dec. 2013.

[7] Y. Zhang, R. Mittra, B.-Z. Wang, and N.-T. Huang, "AMCs for ultra-thin and broadband RAM design," Electronics Letters, vol. 45, no. 10, p. 484, (2009).

[8] R. Mittra and A. Hoenschel, "Some Novel Designs for RFID Antennas and their Performance Enhancement with Metamaterials," 2007 International Workshop on Anti-Counterfeiting, Security and Identification (ASID), (2007).

[9] R. Saad and K. L. Ford, "A triple band Artificial Magnetic Conductor surface incorporating a split ring resonator antenna," 2014 Loughborough Antennas and Propagation Conference (LAPC), (2014).

[10] B. Sanz-Izquierdo, E. A. Parker, J. C. Batchelor and J. A. Miller, "Body armour with integral high impedance surface," Proceedings of the 5th European Conference on Antennas and Propagation (EUCAP), pp. 1061-1064 (2011).

[11] G. K. Palikaras, A. P. Feresidis, and C. G. Parini, "Advances in conformal antennas based on high impedance and EBG metamaterial surfaces," 2011 International Workshop on Antenna Technology (iWAT), (2011).

[12] S. Zhu, K. L. Ford, A. Tennant and R. J. Langley, "Loaded split ring antenna over AMC," in Electronics Letters, vol. 46, no. 14, pp. 971-972, July 2010.

[13] R. Saad and K. Ford, "Miniaturised dual-band artificial magnetic conductor with reduced mutual coupling," Electronics Letters, vol. 48, no. 8, p. 425, 2012.

[14] R. Saad and K. L. Ford, "A miniaturised dual band Artificial Magnetic Conductor using lumped components," Loughborough Antennas \& Propagation Conference, (2011).

[15] B. Sanz-Izquierdo, J. C. Batchelor, and E. A. Parker, "Integration of antennas and high impedance surfaces on ceramic body armor plates," 2011 IEEE International Conference on Microwave Technology \& Computational Electromagnetics, 2011.

[16] Ansoft HFSS, from Ansoft Corporation, Four Station Square Suite 200, Pittsburgh, PA 15219.

[17] C.R. Simovski, P. de Maagt, S.A. Tretyakov, M. Paquay and A.A. Sochava, "Angular stabilisation of resonant frequency of artificial magnetic conductors for TEincidence," Electronics Letters, vol. 40, no. 2, pp. 9293, (2004).

[18] M.E. de Cos, Y. Alvarez and F. Las-Heras, "Novel Broadband Artificial Magnetic Conductor With Hexagonal Unit Cell," IEEE Antennas and Wireless Propagation Letters, vol.10, no., pp.615-618, 2011.

[19] M. Mantash, M.E. de Cos, A. Tarot, S. Collardey, K. Mahdjoubi and F. Las-Heras, "Dual-band textile hexagonal artificial magnetic conductor for WiFi wearable applications," $6^{\text {th }}$ European Conference on Antennas and Propagation (EUCAP), pp.1395-1398, (2012).

[20] M.E. de Cos and F. Las-Heras, "Multi-Band Artificial Magnetic Conductors with high angular stability," $7^{\text {th }}$ European Conference on Antennas and Propagation (EuCAP), pp. 2510-2512, (2013).

[21] M.E. de Cos and F. Las-Heras, "On the advantages of loop-based unit-cell's metallization regarding the angular stability of artificial magnetic conductors", Appl. Phys. A, 118, 699, (2015).

[22] I. Anderson, "On the Theory of Self-resonant Grids" Bell Syst. Techn. J., 1975, 54, pp. 1725-1731.

[23] M. Z. A. Abd. Aziz et al., "Impedance modeling for a unit-cell of the square loop frequency selective surface at $2.4 \mathrm{GHz}$," Antennas and Propagation Conference (LAPC), pp. 161-166, (2013).

[24] H. Fernandez Alvarez, M. E. de Cos Gomez and F. LasHeras, "Angular Stability of Metasurfaces: Challenges Regarding Reflectivity Measurements [Measurements Corner]", IEEE Antennas and Propagation Magazine, vol. 58, no. 5, pp. 74-81, (2016). 